\title{
Preliminary computational study of turbulent flow in the boundary layer of the suction side of an inclined plate
}

\author{
Lukáš Hurda ${ }^{1, *}$ and Richard Matas ${ }^{2}$ \\ ${ }^{1}$ Department of Power System Engineering, University of West Bohemia in Pilsen, Univerzitní 8, 30100 Plzeň, Czech Republic \\ ${ }^{2}$ New Technologies - Research Centre, University of West Bohemia in Pilsen, Univerzitní 8, 30100 Plzeň, Czech Republic
}

\begin{abstract}
A turbulent flow behind a simple thin planar air foil with rounded edges at moderate angle of attack is studied. Reynolds number is about 65000 . First results of CFD analysis are presented, which will be validated with PIV measurements made by Institute of Thermomechanics of the Czech academy of Sciences. All the work is carried out to support the "New theory of flight" presented by Hoffman and Johnson [1]. Large eddy simulation is employed to verify the existence of streamwise vorticial structures and their description. The 3D transient simulation provides more information than the data from a set of PIV measurement planes and it offers also finer time resolution.
\end{abstract}

\section{Introduction}

The new theory of flight presented by [1] suggests methods of description of lift and drag forces that rely on the expected 3D structure of the air foil boundary layer and the wake. The Institute of Thermomechanics of the Czech academy of Sciences has started a project to examine these structures experimentally.

To verify the outcomes of their measurement CFD methods were employed. Most of the data presented in this paper are directly comparable with the experimental results presented in $[2,3,4]$.

\section{Case setup}

The case is a very simple external aerodynamics setup. A flat plate $100 \times 2 \mathrm{~mm}$ with half-cylindrical edges is approached by low turbulent cross flow (TI $=0.5 \%$ ) under $7^{\circ}$ angle of attack. It mimics an experiment with air at $10 \mathrm{~m} / \mathrm{s}$, therefor the flow is modelled as incompressible.

\subsection{Geometry}

The computational domain is a $2 \mathrm{D}$ far field extruded in the spanwise direction. Its shape is designed to ease the creation of spatial discretization mesh.

The distance from aerofoil to outer boundary was derived from Ercoftac UFR 2-05 test case - it is around 20 chords away in all directions, see Fig. 1.

Spanwise extrusion distance was set as long as two chords of the plate, as could be seen in Fig. 2.

The plate is inclined in the stream aligned coordinate system.

\subsection{Boundary conditions}

A no-slip condition is applied on the plate surface and a periodic boundary condition is imposed on the spanwise normal sides of the domain.

On the rest of the outer boundaries, a freestream condition as implemented in OpenFOAM is prescribed. It implies a dual behaviour of each boundary face flux and cell value. For inward fluxes it imposes a globally defined fixed value for velocity and possibly the modelled turbulence variables. For outward fluxes it extrapolates the boundary value. Zero gradient condition for pressure is used in both cases.

\subsection{Computational mesh}

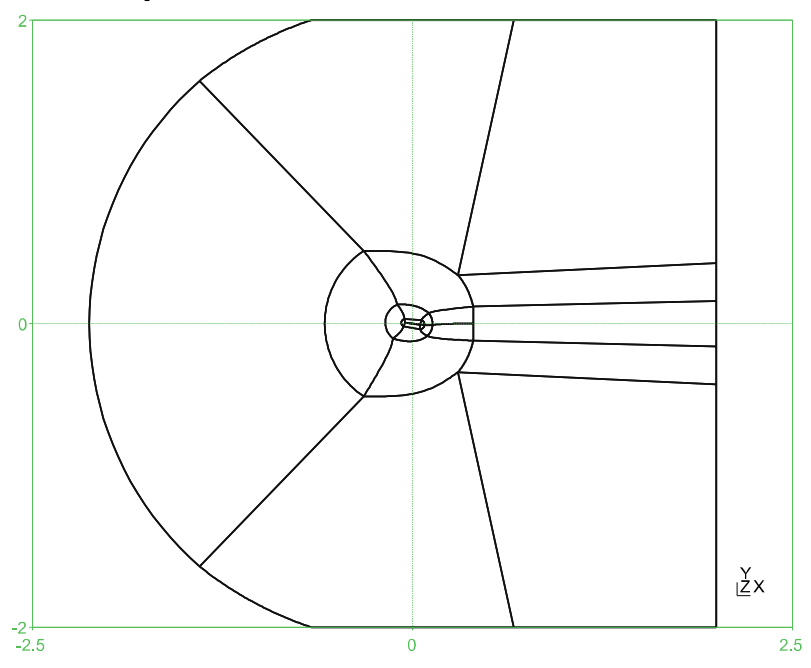

Fig. 1. The blocking structure of the mesh axes ticks in metres while the plate has chord of $0.1 \mathrm{~m}$. 
The spatial discretization is made with a mesh of hexahedral elements arranged in blocks. The blocking around the aerofoil has an $\mathrm{O}$-grid structure. There are three layers of such layout for precise control over cell geometry and buffering the transition into a C-grid structure of the farthest layer. The O-grid copies well the plate with rounded edges while the C-grid allows the mesh to be refined in the region of the wake behind the plate, as seen in Fig. 1.

Further, cut-cell refinements are used inside for arbitrary groups of mesh blocks. The finest mesh uses a double refinement: a spanwise-normal split of the cells of all three $\mathrm{O}$-grid layers plus the far wake region and a full 1-into-8 3D cell refinement of the inner two O-grid layers extended by the wake region of the third O-grid. The utilised models discussed in the next section require the first cell height to lie in the region of nondimensional wall normal direction $y^{+}<1$ and also $\Delta x^{+}<150 ; \Delta \mathrm{z}^{+}<40 ; \quad$ denoting streamwise and spanwise node spacing respectively. These requirements were met with slight reservations. The streamwise direction is finer than required by $\Delta x^{+}$criterion due to high number of cells to cover curvature of leading and trailing edges and to maintain acceptable expansion ratio along the suction and pressure sides. Other mesh quality aspects were tuned in the regions of interest.

The finest mesh used has $2,6 \cdot 10^{7}$ cells.

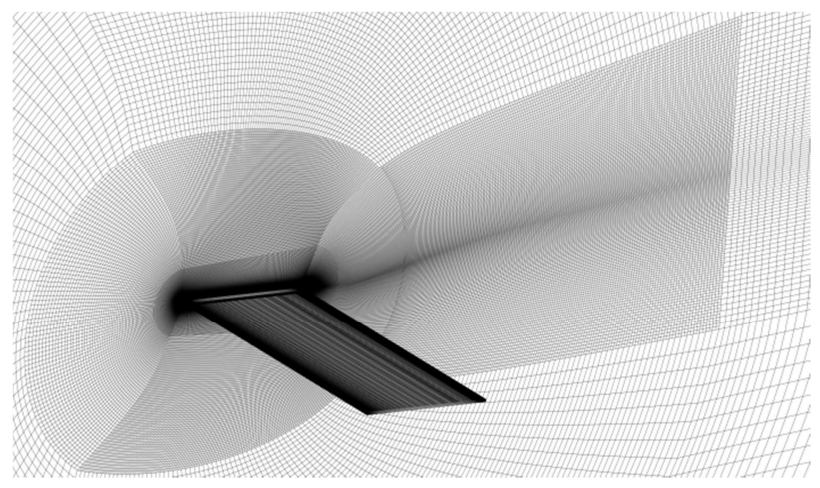

Fig. 2. Detail of the cut-cell refined mesh in the vicinity of the flat plate.

\subsection{Mathematical models}

As the main requirement for this computation is the accuracy of the turbulent behaviour and high spatial and temporal resolution, large eddy simulation was chosen as an appropriate approach.

A modern sub-grid scale turbulence model 'WALE' was used, which should predict the near wall phenomena better than the well-known Smagorinsky model.

Second order implicit time stepping was utilised while keeping the CFL criterion below 0.9. An explicit time discretization scheme is not readily available in OpenFOAM, though it would be beneficial for this case in terms of computation time and resource requirements. The CFL number still needs to be kept lower for high temporal resolution of the small turbulent scales while it is also required for the stability of the employed PISO solver.

\section{The solution process}

Computations were run in parallel in the environment of MetaCentrum or the New Technologies - Research Centre dedicated cluster. 32 cores spread across 84 -CPU nodes using Ethernet network were set as an optimal for feasible parallelization with the $26 \mathrm{M}$ mesh.

The time step for maximum CFL $=0.9$ went as low as $2 \cdot 10^{-6} \mathrm{~s}$.

The flow was initialized using steady potential solution in the first run. It took around $0.15 \mathrm{~s}$ of computational domain time to establish sufficient statistical convergence of the transient solution. This is 15 convective times of the flow passing the aerofoil. Developed flow 3D data were used then directly or remapped on a different mesh where possible.

\subsection{Data sampling}

The 3D data were resampled onto $2 \mathrm{D}$ planes and isosurfaces were computed. The sampling planes correspond to [3] and are annotated in Fig. 3. Mean quantities were obtained with an averaging window of 10 convective times $-0.1 s$ of flow time.

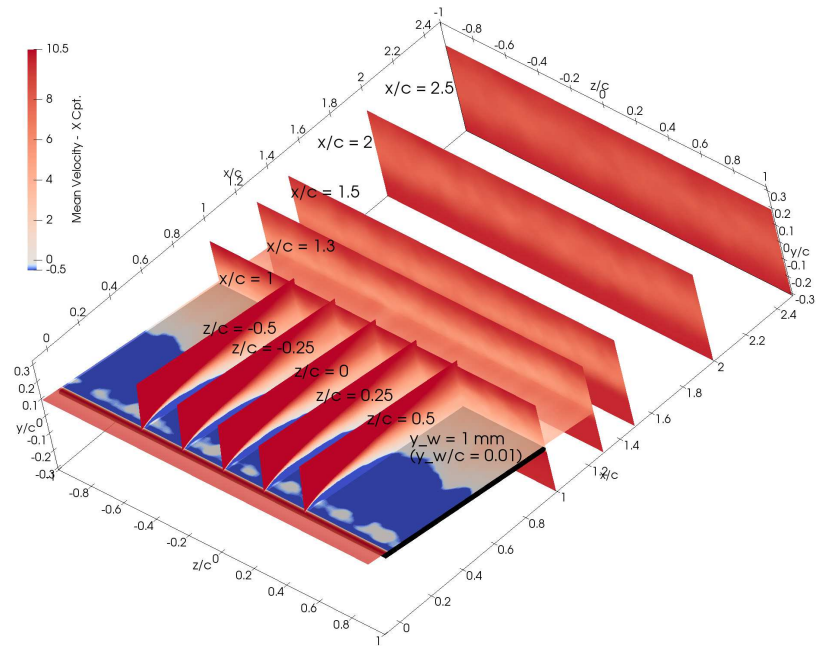

Fig. 3. Sampling planes annotated, mean velocity streamwise component plotted. Plate surface rendered black.

\section{Results}

\subsection{Spanwise-normal cutting planes}

The size and stability of the separation bubble can be assessed from Fig. 4 and 6. A large magnitude of the instantaneous spanwise velocity may be observed in Fig. 4 (up to $80 \%$ of freestream velocity magnitude). The magnitudes of spanwise velocity component in the mean velocity field (Fig. 6) are lower by an order of magnitude, but this still shows the averaging window should be longer, if it will ever be feasible.

\subsection{Suction surface cutting plane}

The vorticial structures above the plate are described using vorticity components contour plots and surface 


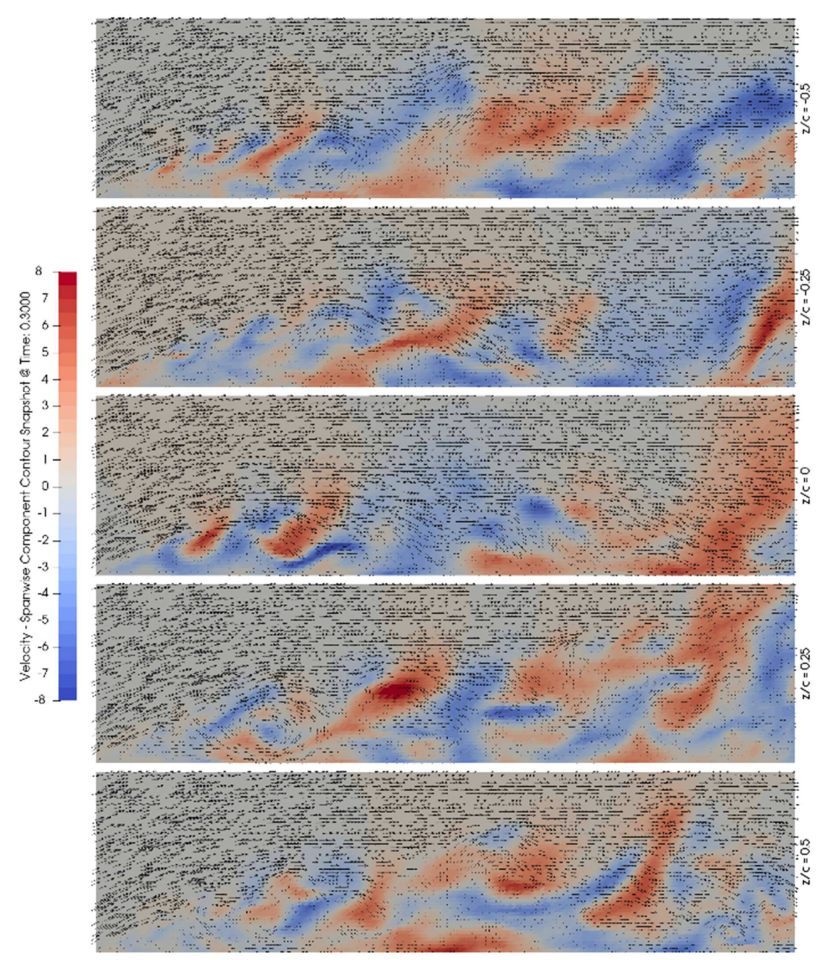

Fig. 4. Spanwise normal planes above plate suction surface. Instantaneous spanwise velocity contours and vectors of instantaneous in-plane velocity.

vector lines in Fig. 5 and 7. Unlike the chaotic behaviour of the instantaneous vector lines, mean vector lines show the bounds of an unstable separation bubble. The instantaneous contours of vorticity give strong evidence of worm-shaped streamwise vortices. Those do not vanish in the averaging process completely, which may be due to their relative stability or due to short averaging window.

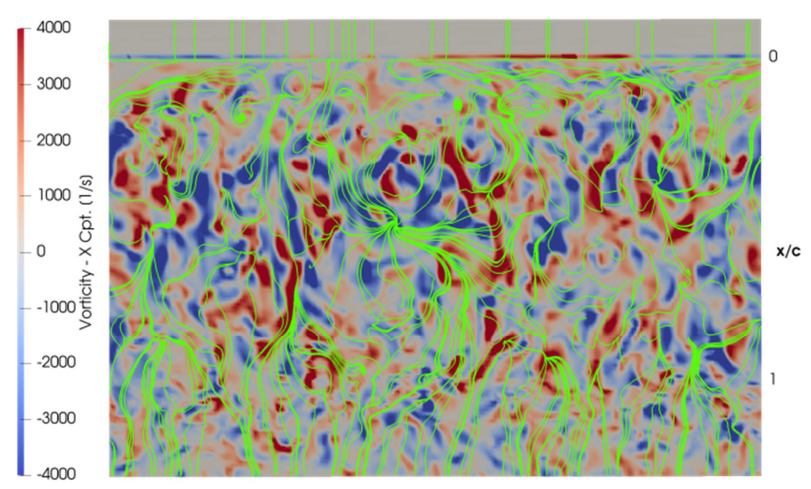

Fig. 5. Plane $y_{w} / c=0.01 \mathrm{~m}$. Instantaneous spanwise vorticity contours and surface vector lines of instantaneous velocity.

The proper orthogonal decomposition (POD) described in [5] was applied to th set of the velocity field snapshots in the plane close above the plate. The snaps were split in 4 spanwise slices, then 2 were used as uncorrelated datasets and two were discarded. This was to take advantage of the long domain span and to get a reasonable amount of snapshots (134 in total, sampled 4 times per one convective time period). The outcomes are presented in Fig. 8 to 10.

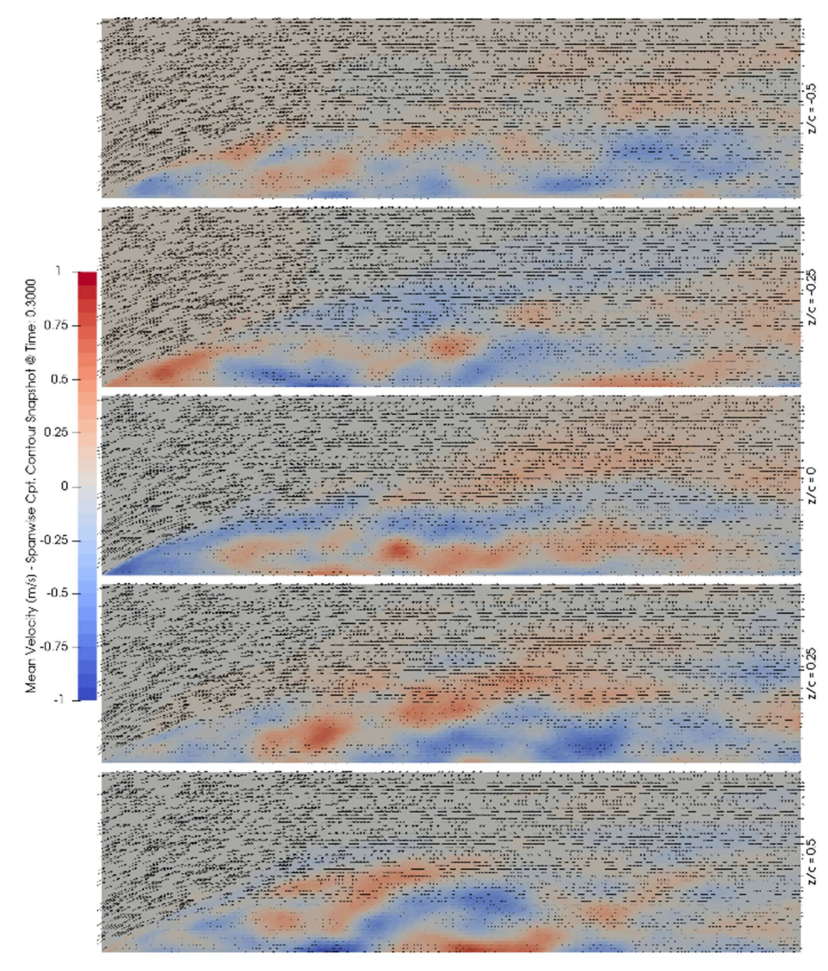

Fig. 6. Spanwise normal planes above plate suction surface. Mean spanwise velocity contours and vectors of mean in-plane velocity.

The strongest modes (Fig. 8) show large structures of the shear layer causing streamwise velocity reduction and notable part of spanwise fluctuations. In mode 3 (right middle part) and most of mode 5, streamwise oriented vortices are clearly visible, even only $1 \%$ chord above the suction surface (see Fig. 10).

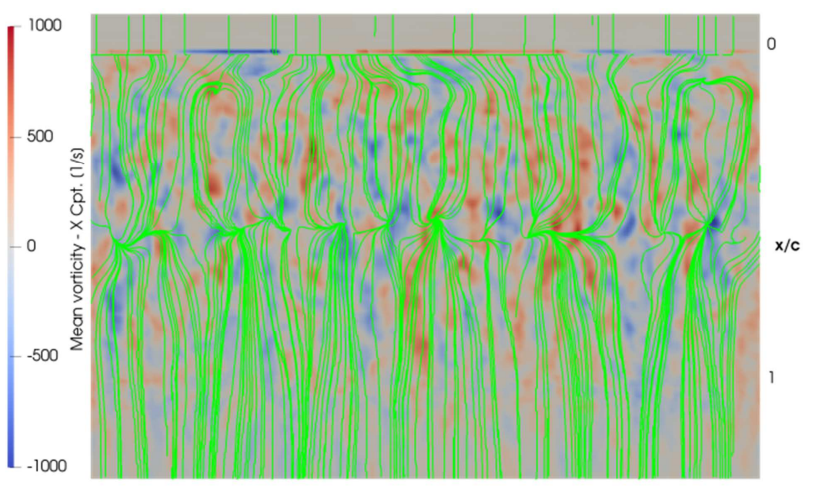

Fig. 7. Plane $y_{w} / c=0.01 \mathrm{~m}$. Mean spanwise vorticity contours and surface vector lines of mean velocity.

Modes 6 and 46 (Fig. 9) are more chaotic, the structures are smaller and the highest dynamical activity takes place in the aft part of the separation bubble, near $x / c=0.6$, and, of course, also in the lowest part of the figure, the zones behind the trailing edge.

Unfortunately, for the vorticity field in the $y_{w} / c=0.01$ plane, the averaging window is not long enough to establish the data for meaningful POD analysis. Even the topology of velocity modes for this cutting plane are not very close to the measurement data published in [4] due to this fact. 

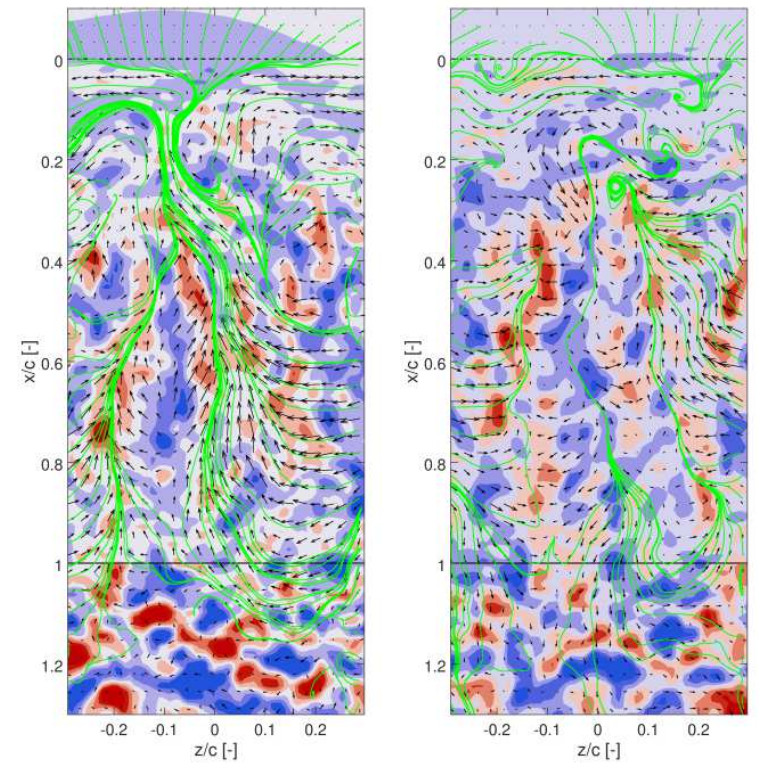

Fig. 8. Spanwise slices of plane $y_{w} / c=0.01 \mathrm{~m}$. Velocity POD modes no. 1 (left) \& 2 (right). Content $3.1 \% \& 2.8 \%$ of TKE.
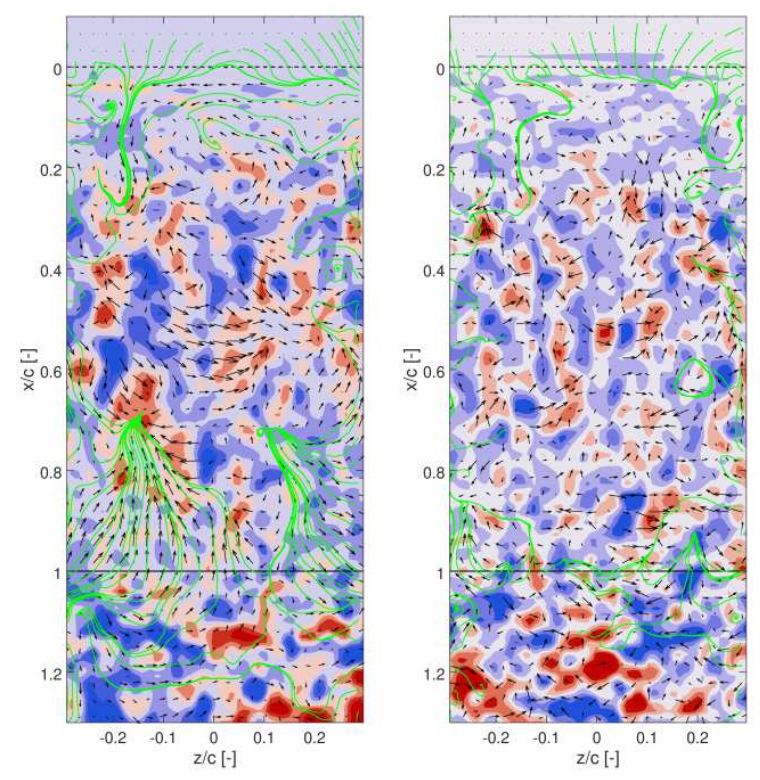

Fig. 9. Spanwise slices of plane $y_{w} / c=0.01 \mathrm{~m}$. Velocity POD modes no. 6 (left) \& 46 (right). Content $2.3 \% \& 1.2 \%$ of TKE.

\subsection{Streamwise normal cutting planes}

In Fig. 11 and 13, the undecomposed velocity fields at streamwise cutting planes are presented. The vertical axis ticks (here the absolute y-coordinate) denote the projections of the trailing edge in the streamwise direction and in the direction oriented along the plate.

The instantaneous field in Fig. 11 exhibits high dynamical activity originating in the suction side boundary layer, which slowly vanishes along the streamwise distance. Eddies of different scales and positions with an axis possibly close to streamwise direction may be distinguished.

The mean field in Fig. 13 has the 3D effects dumped almost completely by the time averaging, even though the averaging window is supposed to be relatively short.
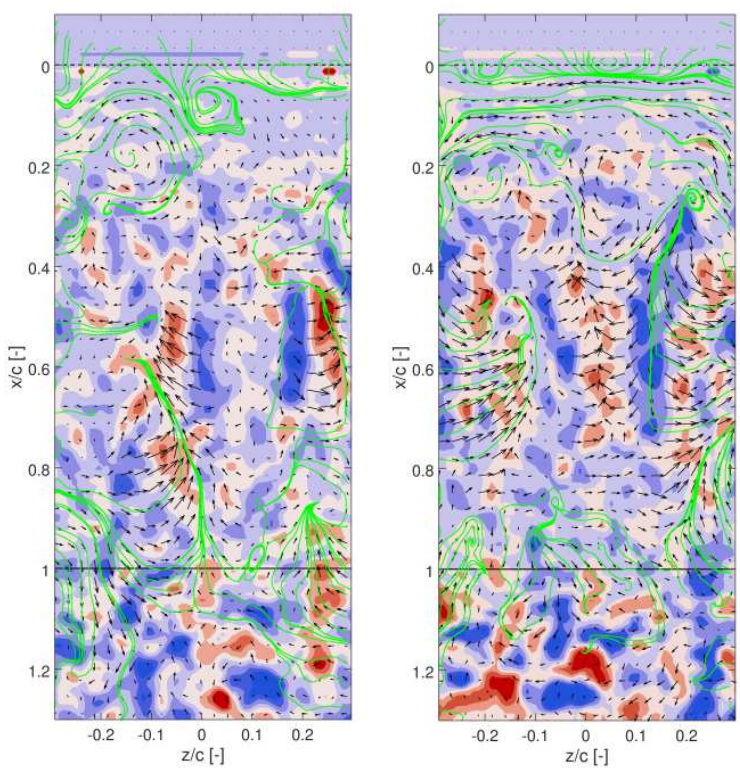

Fig. 10. Spanwise slices of plane $y_{w} / c=0.01 \mathrm{~m}$. Velocity POD modes no. 3 (left) \& 5 (right). Content $2.5 \% \& 2.3 \%$ of TKE.

This is a good premise for the POD. The downwash effect is also clearly observable in the mean field.

Fig. 12 and 14 show the selected POD modes of velocity in the streamwise planes. Same spanwise slicing of the planes as described in paragraph 4.2 is applied. Only the first 3 planes behind the trailing edge are shown. The dashed line shows the normal projection of the trailing edge.

The dominant mode no. 1 contains wide streaks of decreased and increased velocity with a vortex between them. Streaks of width around $0.3 c$ relate to findings presented in [2].

The higher modes present similar behaviour, but in a smaller scale and more chaotic patterns. The mode no. 17 gets close to the 'two row checkerboard' vortex pattern also visible in higher modes described in [4].

\subsection{Vorticity iso-surfaces}

Comparing Fig. 15 and 16, the damping of $3 \mathrm{D}$ vorticity is clear. The 3D CFD data are much more reliable source for such renders than the reconstruction of the 2D3C measurements. When the time averaging window would be prolonged, the mean vorticity iso-surface will hopefully get closer to be prismatic in the spanwise direction.

\section{Conclusion}

The turbulent flow around a flat plate was numerically computed. It shows similar 3D structures as the experiment, especially when comparing the streamwise normal planes with [2], commented in paragraph 4.3.

The suction side boundary layer cutting plane needs longer computation time to establish sufficient mean field. 


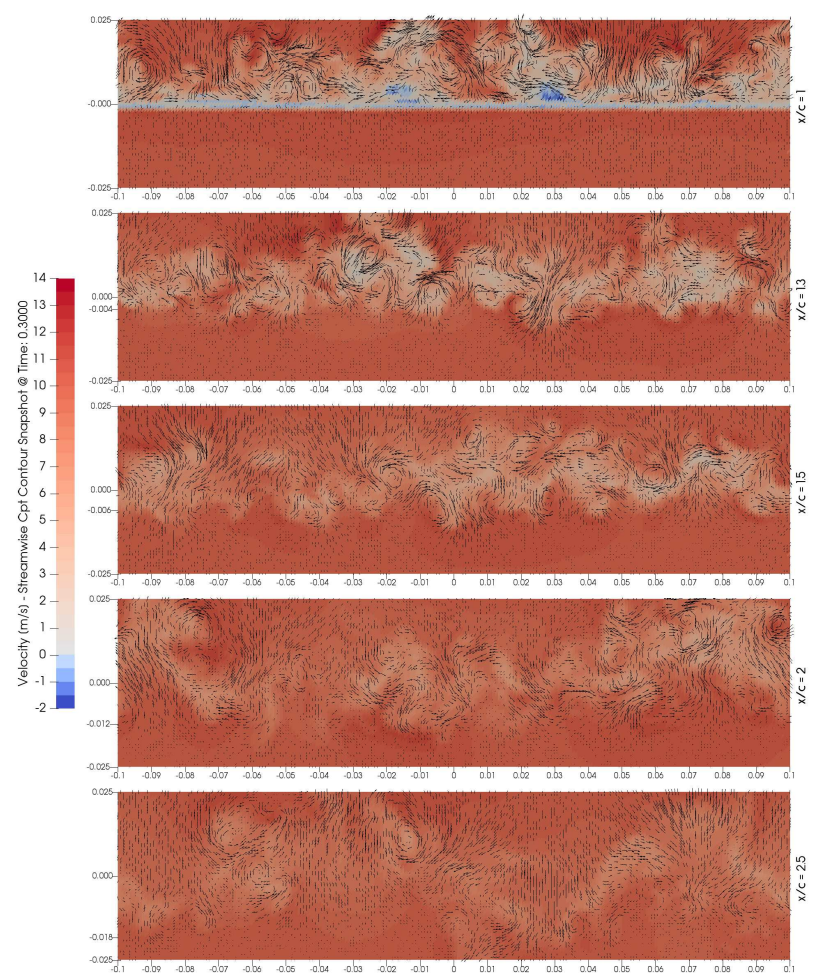

Fig. 11. Streamwise normal planes - slices through the wake. Instantaneous spanwise velocity contours and vectors of instantaneous in-plane velocity.
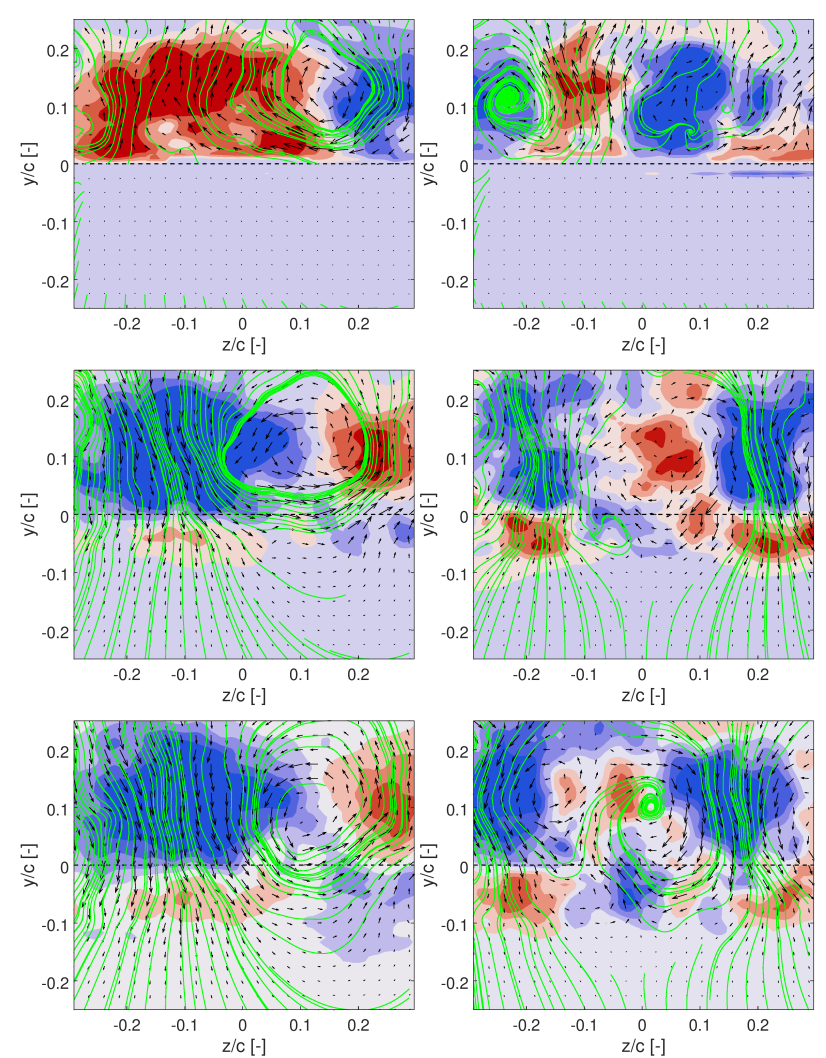

Fig. 12. Parts of streamwise normal planes $\mathrm{x} / \mathrm{c}=1,1.3,1.5$ (top to bottom). POD modes of velocity no. 1 (left) \& 4 (right).

Content $\sim 4.6 \% \& \sim 3.2 \%$ of TKE.

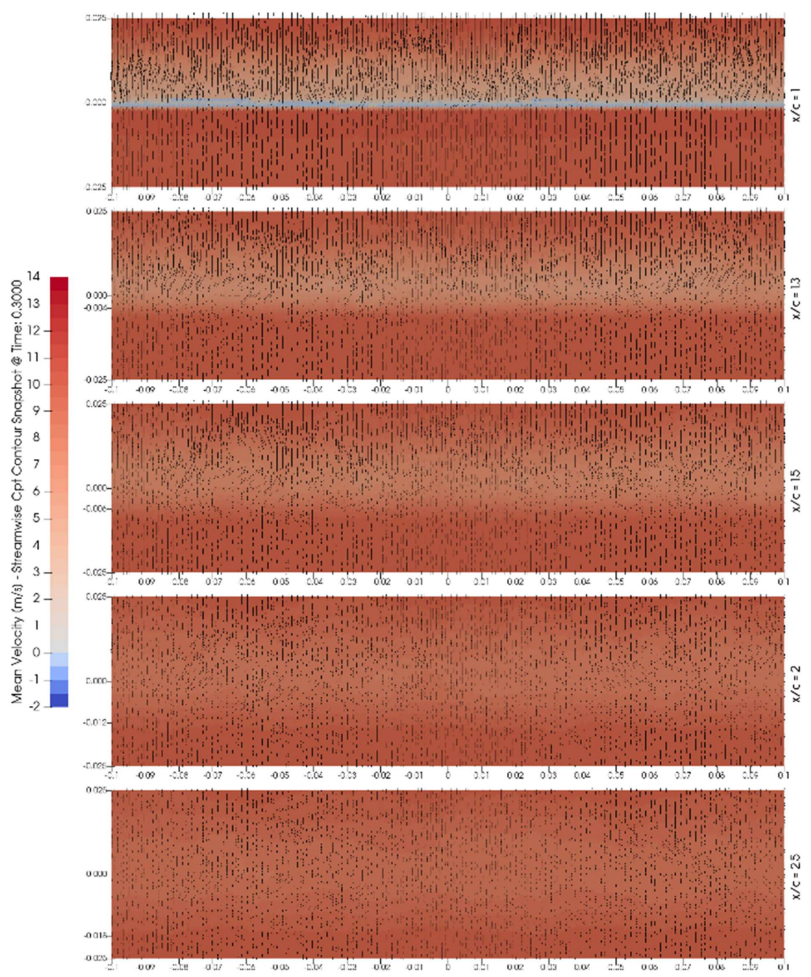

Fig. 13. Streamwise normal planes above plate suction surface. mean spanwise velocity contours and vectors of mean in-plane velocity.
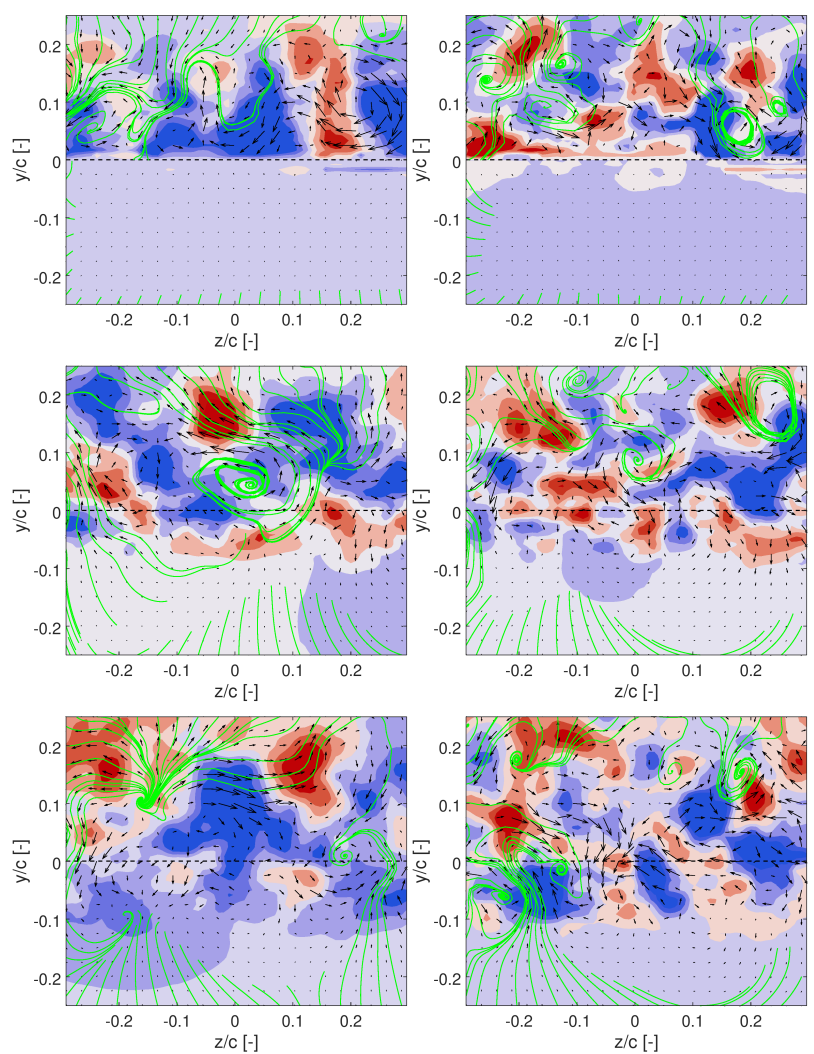

Fig. 14. Parts of streamwise normal planes $\mathrm{x} / \mathrm{c}=1,1.3,1.5$ (top to bottom). POD modes of velocity no. 7 (left) \& 17 (right). Content $\sim 2.5 \%$ \& $\sim 1.8 \%$ of TKE. 
More work is needed to address the issue with an unexpected behaviour of the flow. The separation region on the suction side is significantly larger than observed by PIV. A sensitivity analyses concerning angle of attack, changes in Reynolds number or subgrid-scale turbulence model are necessary.

Future work may also concentrate on the implementation of the POD in $3 \mathrm{D}$ fields and possibly other spatio-temporal decomposition methods used by prof. Uruba.

For the purpose of lowering computational resources demand, upcoming computations will be carried out with the external flow velocity lowered to $5 \mathrm{~m} / \mathrm{s}$. Extensive experimental work is being done at the side of Czech Academy of Sciences also using such boundary condition.

This work was supported by the Grant Agency of the Czech Republic, project No. 17-01088S and by the project of specific research UWB SGS-2016-045. Access to computing and storage facilities owned by parties and projects contributing to the National Grid Infrastructure MetaCentrum provided under the programme "Projects of Large Research, Development, and Innovations Infrastructures" (CESNET LM2015042), is greatly appreciated.

\section{References}

1. J. Hoffman, C. Johnson, The Mathematical Secret of Flight, Normat 57, 4, pp.1-25, (2009)

2. V. Uruba, D. Pavlík, P. Procházka, V. Skála, V. Kopecký, On 3D flow-structures behind an inclined plate, EPJ Web of Conferences, 143, article number 02137 (2017)

3. P. Procházka, V. Uruba, V. Skála, On the 3D structure of the flow-field in the vicinity of inclined plate, (to be published)

4. V. Uruba, P. Procházka, V. Skála, On 3D Flow Structure of the Boundary Layer on the Suction Side of a Plate, EPJ Web of Conferences, 180, article number 02112, 2018.

5. V. Uruba, Decomposition methods in turbulence research, EPJ Web of Conferences, 25, article number 01095, 2012.

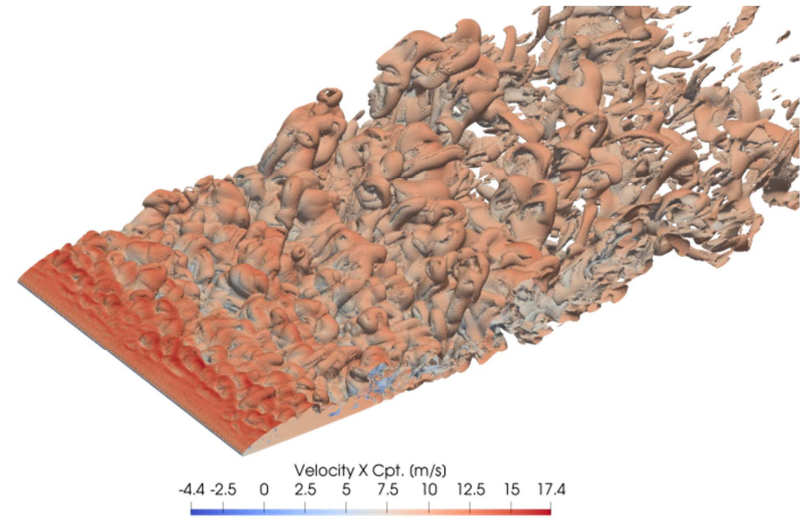

Fig. 15. Iso-surface of instantaneous vorticity $500 \mathrm{l} / \mathrm{s}$. Coloured by streamwise velocity.

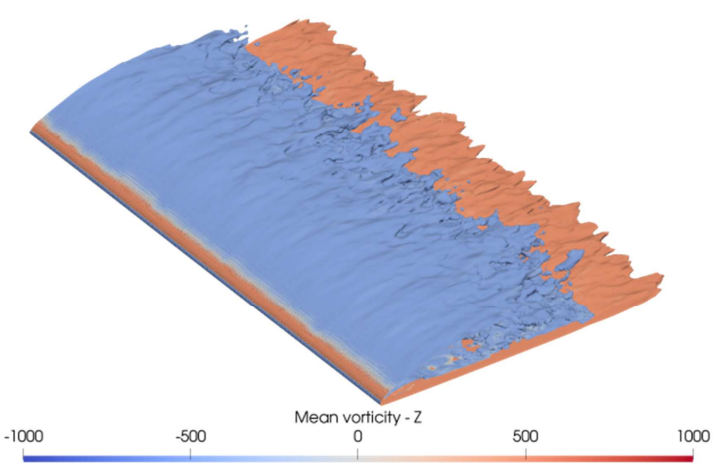

Fig. 16. Iso-surface of mean vorticity $500 \mathrm{l} / \mathrm{s}$. Coloured by its spanwise oriented component. 